\title{
NUMERICAL SIMULATION OF SHOCK-INDUCED SEPARATED FLOWS IN OVEREXPANDED ROCKET NOZZLES
}

\section{A. Shams, S. Girard, and P. Comte}

\author{
Institut Pprime \\ UPR CNRS 3346, ENSMA \\ Université de Poitiers \\ CEAT, Route de l'Aérodrome 43, Poitiers F-86036, France
}

Flow separation in rocket nozzles is undesirable because of its unsteady and nonsymmetric nature, which leads to dangerous side-loads. At the initial stages of start-up, when a thrust optimized contour (TOC) nozzle operates under overexpanded conditions, free shock separation (FSS) takes place. Under certain conditions, this free separated flow reattaches back to the nozzle wall and forms restricted shock separation (RSS). The appearance of restricted shock separated flow depends upon the nozzle contour in a well-defined range of nozzle pressure ratios (NPR) and is characterized by a cap-shock pattern. The flow transition process from FSS to RSS flow configurations is a complex phenomenon and has been an area of interest for a few decades now. In the present study, an attempt has been made to understand the formation of the cap-shock pattern and the RSS flow configuration in a thrust optimized contour (TOC) nozzle. The presented research work consists of two parts. In the first part of the paper, numerical investigation of flow transition (FSS $\longrightarrow$ RSS) has been performed to understand the formation of the cap-shock pattern, which is believed to be the main cause for this flow transition from FSS to RSS. Axisymmetric numerical calculations on a wide range of NPRs (15-25) are performed to reproduce the forward transition process and are found to be in good agreement with the experiments. In the second part, some light has been shed on various aspects of RSS flow regime. Three-dimensional (3D) numerical simulations have been performed on a wide range of NPRs, i. e., 25.0, 30.0, 38.0, 41.0, and 46.0. Detailed analysis of these numerical results allows examining the evolution of the separation point and the cap-shock pattern with respect to the NPR. Furthermore, some insights based on the axial momentum along the nozzle axis and radial momentum distributions across the quadruple point are given.

This is an Open Access article distributed under the terms of the Creative Commons Attribution-Noncommercial License 3.0, which permits unrestricted use, distribution, and reproduction in any noncommercial medium, provided the original work is properly cited. 


\section{INTRODUCTION}

Flow separation in rocket nozzles has been an area of interest over the last few decades. Several experimental, theoretical, and numerical studies are performed to understand this separation phenomenon in rocket nozzles. Flow separation in rocket nozzles is considered undesirable due to its unsteady and nonsymmetric nature which causes lateral forces, known as side loads, which may cause structural damage. Extensive investigations of flow separation in conical rocket nozzles, running under overexpanded conditions, were performed at the Jet Propulsion Laboratory (JPL) during the late 1940s and early 1950s [1]. It was reported that when the nozzle-wall pressure at the nozzle exit was lowered by more than about 0.4 times the ambient pressure, flow separation takes place. Due to this separation, the wall pressure quickly rises to a plateau (slightly lower than the ambient pressure). This sudden rise in wall pressure is because of the separation shock formed by the strong change in pressure. Once the flow was separated, no reattachment occurred: that is why this type of flow separation is known as FSS.

Later on, in 1973, the work of Nave and Coffey [2] on the J-2S engine demonstrated that, apart from FSS flow regime, there exists another type of flow separation, known as RSS. The RSS is a complex flow regime, and a number of experimental and numerical studies in the past have been performed to understand the phenomenology of the flow [2-20]. The RSS flow regime was observed only in TOC [2] and, more recently, in compressed truncated perfect (CTP) nozzles [16], i. e., in nozzles involving an internal shock in the full flowing regime. Restricted shock separated flow is characterized by a small recirculation pocket followed by reattachment on the nozzle wall. The main flow involves a special shock pattern called cap shock, followed by a trapped vortex surrounded by an annular supersonic jet. Existence of reversed flow (trapped vortex) in the plume of these nozzles, which was first reported by Chen et al. [3], has been confirmed by several experimental [21] and numerical [15, 20] investigations. It is believed that this trapped vortex is linked with the cap-shock pattern. With axisymmetric Reynolds-averaged Navier-Stokes (RANS) calculations on a TOC nozzle, Frey and Hagemann [7] have shown that this cap shock can be interpreted as the inverse Mach reflection of the internal shock which emanates from the region close to the nozzle throat. A 3D unsteady numerical simulation of the flow in a TOC nozzle has been performed by Deck et al. [10, 15], but their study was mainly devoted to the hysteresis of the transition between the FSS and RSS regimes and to the evaluation of side loads. Shimizu et al. [16] investigated flow transition from FSS to RSS in a CTP nozzle. In the RSS flow regime, they were not able to detect the weak internal shock which interacts with the Mach disk but observed a small low-expansion region attached to the Mach disk.

A number of studies in the past have been performed to find out the origins of the side loads $[2,4,5,7-11,13-15,17]$. It is proven that in TOC nozzles, a major side-load peak occurs as a result of transition of the separation pattern from FSS 
to RSS (FSS $\rightarrow$ RSS) [7, 13]. The generation of large side loads in the rocket nozzle during the start-up transient can induce serious launch problems and may also destroy the engine hardware in sea-level tests. In addition to large side loads, high thermal stresses on the nozzle wall can be generated during the transient of the operation, because of the asymmetric behavior of the separation line and, in the case of RSS, the reattachment of the separated flow. Transition from FSS to RSS is a complex phenomenon and has been an area of interest for the last few decades. Various experimental and numerical studies have been performed to understand this phenomenon $[2,4,5,7-11,13-15,17]$ which, nevertheless, requires further clarification. Nave and Coffey [2], reported a hysteresis loop to occur in the case of the J-2S nozzle. Later on, Chen et al. [3] performed numerical calculations for the J-S2 nozzle and were able to capture the hysteresis flow field for a wide range of NPRs. Reasons for the transition between the separation patterns have been discussed by Hagemann et al. [11] who identified the capshock pattern to be the cause of this transition.

In spite of various attempts to understand the flow separation in rocket nozzles, the question about the formation of cap-shock pattern is still open and needs to be explored. In this first part of the paper, the attention was focused on the formation of the cap-shock pattern and its consequences. Hence, numerical simulations are performed on a wide range of NPRs to achieve flow transition from FSS to RSS regime. These simulations, presented in section 4, are performed on a TOC nozzle, which is experimentally investigated at Laboratoire d'Etudes Aérodynamiques (LEA, France, recently merged into the Institut Pprime) and is denoted as LEATOC nozzle [8].

In section 5, a detailed study of RSS flow regime in the same LEATOC nozzle has been carried out in three dimensions, in Detached-Eddy Simulation [20], with emphasis on the following points:

- phenomenology of RSS and cap-shock pattern which is the main cause for RSS;

- visualization of flow discontinuities with the help of shock and compression functions $[16,22]$

- behavior of internal shock (that appears near the throat of the nozzle) at different NPR;

- length of the cap-shaped oblique shock with respect to the separated incident oblique shock with the variation of the NPR;

- size and behavior of the trapped vortex that appears behind the cap-shock pattern and its 3D effects; and

- how the size of the recirculation bubble (that appears due to the reattachment of the separated flow) varies with the NPR. 


\section{PHENOMENOLOGY OF SEPARATED FLOWS}

An adverse pressure gradient of sufficient strength can cause the boundary layer to separate. Such a condition typically occurs in the rocket nozzle when it operates under overexpanded condition (when the ratio of nozzle wall pressure inside to the ambient pressure is less than 1). Past research has made it clear that two different separation patterns exist: the classical FSS and RSS [2].

\subsection{Free Shock Separation}

In the case of FSS, the overexpanded flow fully separates from the wall. The resulting streamwise mean wall pressure evolution is mainly governed by the physics of the shock-wave/boundary-layer interaction. The separation and the subsequent formation of the recirculation zone give rise to an oblique shock wave near the wall, as depicted in Fig. 1. This incident oblique shock interacts with the Mach disk (or stem) and consequently forms a reflected shock. This common point of interaction is called a "Triple Point" (TP). The separated jet flow continues as a free jet. Fluid outside the nozzle is sucked into it and separates from the nozzle lip, yielding a counterrotating recirculation bubble with respect

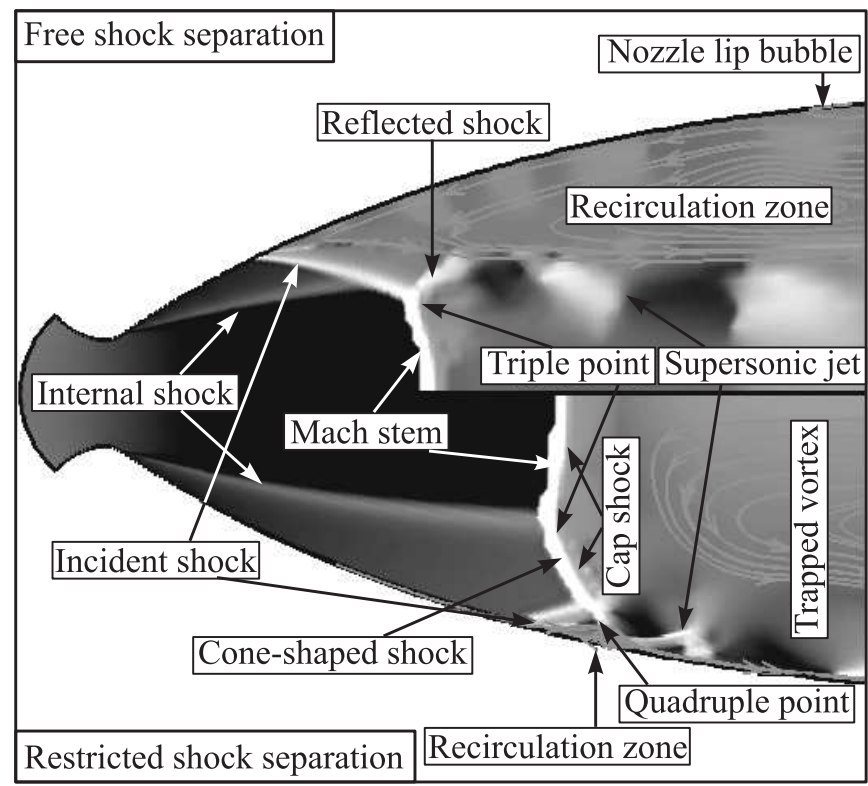

Figure 1 Free shock and restricted shock separation flow regimes 
to the massive recirculation zone. The exhaust jet flow is delimited by a mixing layer through a channel. This mixing layer can be considered as a "fluidic wall" in which the supersonic jet is confined.

\subsection{Restricted Shock Separation}

The appearance of the restricted shock separated flow regime depends upon the nozzle contour in a defined range of NPRs. This special type of separated flow exists only in the nozzles which contain an internal shock, which appears near the throat region. This internal shock interacts with the normal shock (Mach stem, MS) far away from the throat. Consequently a cone-shaped reflected shock (CSS) forms from this common point of interaction. This point of interaction is called TP. A quadruple point (QP) exists where the reflected cone-shaped shock interacts with the incident oblique shock, forming two reflected shocks. As a result, formation of cap-shock pattern takes place. A sketch of the cap-shock pattern is shown in Fig. 2. A free shear layer and the separated boundary layer interact with these reflected oblique shocks and consequently form two expansion waves. Downstream of the interaction, the boundary layer reflects back towards the nozzle wall and reattaches, forming a small recirculation zone attached to the wall. A large stabilized adverse pressure gradient vortex appears downstream of the cap-shock pattern surrounded by annular supersonic jets.

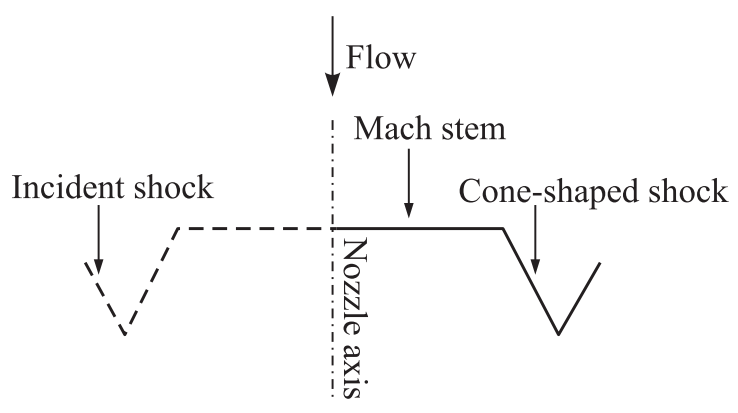

Figure 2 Sketch of cap-shock pattern

\section{DESCRIPTION OF THE COMPUTATIONS}

All the numerical calculations are performed on a TOC nozzle experimentally investigated at LEA [8] and denoted as LEATOC nozzle. This LEATOC nozzle is a thrust optimized contoured nozzle which is approximated by a parabola following Rao's method [23]. The LEATOC nozzle has a throat radius $r_{t}=0.01362 \mathrm{~m}$, 
divergent length $L=0.204 \mathrm{~m}$, and an area ratio $\epsilon=A_{\text {exit }} / A_{\text {throat }}$ of about 30.32 . The computational domain used to perform numerical simulations is set according to the experimental setup [14]. It is composed of two zones, i. e., (i) nozzle, and (ii) exterior, as shown in Fig. 3. The computational domain outside of the nozzle is extended 8 and 2 times the nozzle exit radius in axial and radial directions, respectively.

The most relevant Reynolds number is, certainly, the Reynolds number based upon the boundary-layer thickness upstream of the separation, which is a priori unknown. The

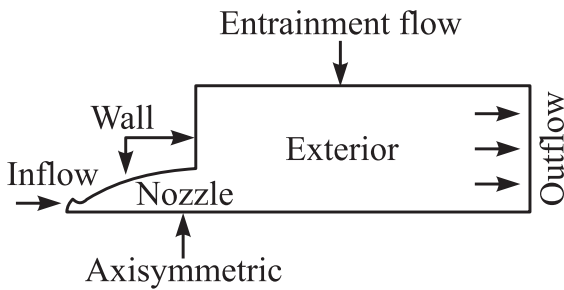

Figure 3 Meridian section of the computational domain with prescribed boundary conditions throat Reynolds number $R_{e_{t}}$ is easier to comprehend. With the aid of one-dimensional (1D) isentropic arguments, one can work out $R_{e_{t}} \approx 0.45 \mathrm{NPR} \cdot 10^{5}$, with the above numerical value of $r_{t}$.

The computational domain is limited by the nozzle wall (and the symmetric axis for an axisymmetric simulation). A subsonic inflow boundary is prescribed in a cross section located in the convergent part at a small distance upstream of the throat; a solid wall is used in the outer part of the nozzle exit plane and some freely chosen external and downstream boundaries are prescribed. By assuming that the upcoming flow is free from any perturbations, the nozzle inlet can, therefore, be considered as steady (except, of course, within the incoming turbulent boundary layer at the nozzle wall). A cold driving gas is prescribed at the nozzle inlet. This nozzle inlet boundary is divided into two segments:

(1) an inviscid segment with subsonic inflow on which 5 quantities are prescribed: the stagnation pressure and temperature $\left(T_{t}=300 \mathrm{~K}\right)$, the direction of the velocity $\tan ^{-1}(V / U)$ orthogonal to the boundary, the turbulent kinetic energy $k=10^{-2} \mathrm{~m}^{2} / \mathrm{s}^{2}$, and the specific dissipation $\omega=500 \mathrm{~s}^{-1}$; and

(2) a boundary-layer segment of thickness $0.1 \mathrm{~mm}$ on which only nondimensional profiles of velocity $u / u_{\infty}$, temperature $\left(T-T_{\text {wall }}\right) /\left(T_{\infty}-T_{\text {wall }}\right)$, turbulent kinetic energy $k / u_{\infty}^{2}$, and dissipation $\epsilon \mu /\left(\rho u_{\infty}^{4}\right)$.

At the walls, a nonslip adiabatic condition is set, with prescribed wall temperature $T_{\text {wall }}=300 \mathrm{~K}$. For external boundary conditions, the LODI (Locally One-Dimensional Inviscid) method, introduced by Poinsot and Lele [24], is used. In order to reduce the reflection of pressure waves at the downstream boundary where the static pressure is imposed, an approach proposed by Rudy and Strikwerda [25] has been used, in which the amplitude of the incoming wave 
is linked to the difference between the local pressure $P$ and the atmospheric pressure $P_{a}$.

An entrainment inflow, i. e., flow entering into the computational domain, is imposed by five quantities, the stagnation pressure and temperature $\left(T_{t}\right.$ $=300 \mathrm{~K}$ ), direction of velocity normal to the boundary, ambient turbulent kinetic energy level $k=10^{-4} \mathrm{~m}^{2} / \mathrm{s}^{2}$, and specific dissipation $\omega=500 \mathrm{~s}^{-1}$. The chamber temperature $(300 \mathrm{~K})$ was set equal to the ambient temperature. The computation starts with either an impulsive or a sequential start-up process. For the impulsive start-up process, the initial condition is quiescent everywhere, except that the chamber condition is prescribed up to the nozzle inlet. For sequential start-up process, the initial flow field is the flow field previously computed along with the new prescribed chamber pressure at the inflow location.

Three sets of mesh in the axisymmetric configuration have

Table 1 Types of meshes used for the grid sensitivity analysis

\begin{tabular}{ccrc}
\hline Nozzle & Exterior & \multicolumn{1}{c}{ Total } & Type \\
\hline $160 \times 100$ & $160 \times 180$ & 44,800 & $A$ \\
$200 \times 120$ & $200 \times 180$ & 60,000 & $B$ \\
$300 \times 180$ & $200 \times 240$ & 102,000 & $C$ \\
\hline
\end{tabular}

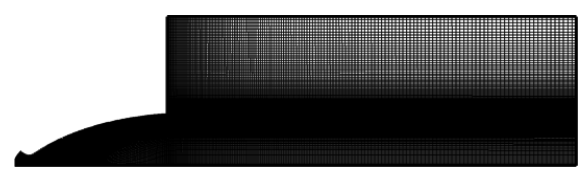

Figure 4 Mesh of the computational domain been tested in order to check the grid sensitivity. Their characteristics are given in Table 1. The first one, i.e., mesh type A, contains $160 \times 100$ grid points inside the nozzle and yields a minimum grid space (first grid point) normal to the nozzle wall equal to $10^{-6} \mathrm{~m}$. The dimensionless distance $r^{+}$, which is a Reynolds number based on the friction velocity $u_{\tau}$, is given as

$$
r^{+}=\frac{\sqrt{\rho_{w}\left|\tau_{w}\right|}}{\mu_{w}} r=\frac{r u_{\tau}}{\nu_{w}} .
$$

The value of $r^{+}$based on RANS (Wilcox $k-\omega$ ) model calculations was found to be less than 2 for this case. This study of grid sensitivity was done on free shock separated flow regime at NPR $=16.5$. The results obtained show no subtantional difference with regard to the global flow structure inside the nozzle, except a better capture of thin boundary layer profiles for the meshes of types $B$ and $C$ with the value of $r^{+}<1$, which is satisfactory for the correct calculation of the viscous stresses. For the present study mesh type $B$ is considered (as shown in Fig. 4), which contains around 10 to 30 grid points inside the boundary layer and the first mesh point is located at $10^{-7} \mathrm{~m}$ from the nozzle wall. 


\section{FORMATION OF THE CAP-SHOCK PATTERN}

\subsection{Numerical Strategy}

Numerical study on LEATOC nozzle has been performed by using the TGNS3D code developed at CEAT/LEA (Poitiers, France). This code solves the 3D unsteady compressible Navier-Stokes equations on multiblock structured grids. The Navier-Stokes equations are discretized in space by using a cell centered finite volume method. For this part of the paper, Wilcox standard $k-\omega$ model [26] with realizability correction [6] has been used for the flow prediction. Roe's flux difference splitting scheme is employed to obtain the flux at the cell interface. The monotone upstream-centered schemes for conservation laws (MUSCL) approach has been used to extend the spatial accuracy to second order with the combination of minmod limiter, to prevent numerical instabilities in shock regions. A diagonally dominant alternating direction implicit (DDADI) approach has been used for the inversion of a large sparse matrix system for the implicit scheme.

A wide range of NPR is investigated by using axisymmetric URANS calculations in order to reproduce the transition process from FSS to RSS. For this purpose, stagnation to ambient pressure ratio $P_{0} / P_{a}$, i.e., NPR, is increased from 15 to 25 with unitary staircase increments $\triangle \mathrm{NPR}=0.5$ and the time elapsed at each $\triangle \mathrm{NPR}$ is $20 \mathrm{~ms}$ until the critical NPR (CNPR) is achieved. This time elapsed (i. e., $20 \mathrm{~ms}$ ) for each constant NPR corresponds to around 8 times the convective time based on $U_{t}$ (velocity at nozzle throat, $\approx 330 \mathrm{~m} / \mathrm{s}$ ) and $L_{d}$ (length of the computational domain, $\approx 0.8 \mathrm{~m}$ ) which is sufficient to capture at least 10 successions of shock excursion and thus any sudden change in the flow structure that could occur during the transition process. Once CNPR is reached, calculations are performed till the RSS flow regime is observed.

\subsection{Results and Discussion}

The cap-shock pattern has been considered as one of main causes for the flow reattachment (i. e., restricted shock separated flow regime) in rocket nozzles, in a well defined range of NPR. Such a condition occurs in TOC type nozzles, in which an internal shock appears near the throat region. The experimental study performed by Nguyen et al. [14] on the LEATOC nozzle has shown that during the start-up process of this nozzle at low NPR, there exists FSS flow regime. This free shock separated flow suddenly turns into RSS flow regime when NPR reaches the value of 24 . Following this, axisymmetric flow transition has been performed to understand the flow physics and main elements which cause this sudden change in the flow behavior. Calculations are performed in a 
wide range of NPR starting from NPR $=15$ (pure FSS flow regime) to 25 (RSS flow regime). Results and discussion on various aspects of the cap-shock pattern formation based on successfully reproduced flow transition (from FSS to RSS) at $\mathrm{NPR}=24$ are presented here.

The evolution of flow structure during the whole flow transition process is beyond the scope of this paper. Here, the focus is on the formation of cap-shock formation. Hence, a few snapshots are selected to show the evolution of flow. Free and restricted shock separation flow regimes contain a variety of flow discontinuities. Hence, a pseudo shadowgraph (isocontours of the density Laplacian $\left.\partial^{2} \rho / \partial^{2} x+(1 / r)(\partial / \partial r)(r(\partial \rho / \partial r))\right)$ is used for fine flow structure detection.

Figure $5 a$ shows the successfully reproduced FSS regime at NPR $=16.5$. An oblique shock is formed due to the flow separation, which interacts with the

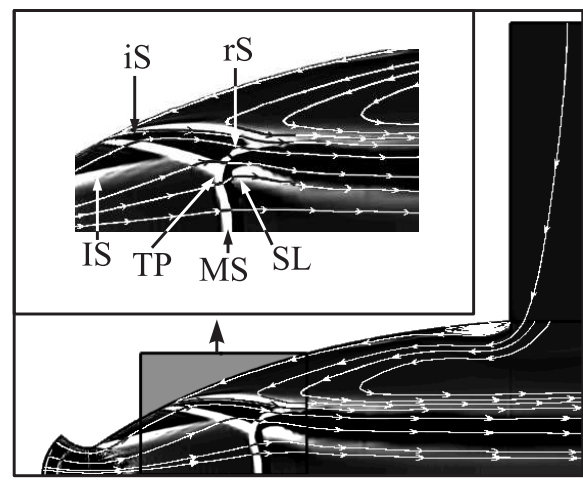

(a)

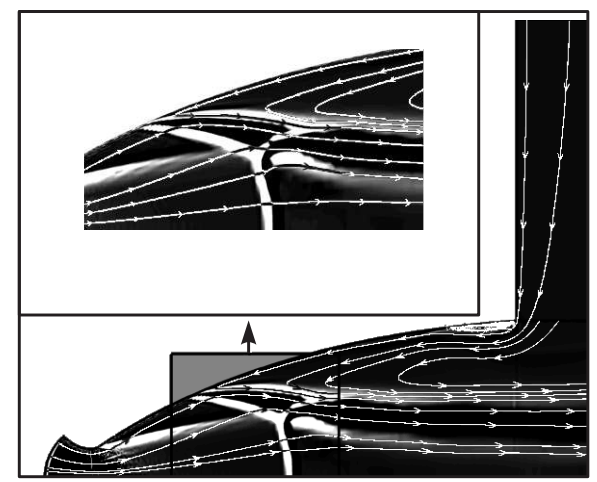

(b)

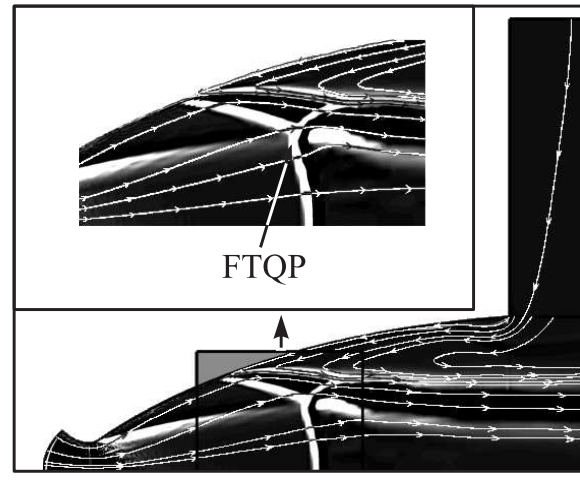

(c)

Figure 5 Time-averaged shadographs and streamlines at NPR: (a) 16.5; (b) 19.5; and $(c) 24.0$ 
Mach disk (MD), here it is called an MS. A reflected shock appears as a result of this interaction, and the point of interaction is called TP. On the other side, the internal shock which forms near the throat of the nozzle interacts with the incident oblique shock from the wall. This interaction causes a small deflection in the oblique shock. A zoom near the TP, internal and the incident shock clearly shows this deflection. A slip line emanates from TP which is inclined towards the nozzle axis and also diverts the flow, emphasized by the streamlines.

As depicted in Fig. 5, with the increase in NPR, separation point and the MS respectively move downstream. As a consequence of this shift in the separation point location, the interaction point of the internal shock on the incident shock moves towards the TP. Figure $5 c$ shows a snapshot when this internal shock reaches the TP. To the best of the present authors' knowledge, no such type of interaction has been reported or seen earlier in literature. It is interesting to note that a clean curved incident shock now interacts directly with the MS along with the internal shock and a slight change in the angle of reflected shock has been observed after this very interaction. It seems that the resulting reflected shock may be the common result of internal and incident shock interaction with the MS. Looking at Fig. 6, one can notice that the flow behavior after this interaction seems to change into the RSS flow regime. Hence, this point of interaction (a common point for MS, internal, incident, and the reflected shocks) is called "Flow Transition QP (FTQP)." It is worthwhile to mention here that this FTQP appears only in the nozzles in which an internal shock appears near the throat region (see Fig. $5 c$ ). It has been observed that this FTQP point appears when the NPR is about 24, and experimentally it is shown that in LEATOC nozzle, flow transition takes place when $\mathrm{NPR}=24[13,14]$. One may conclude that the NPR at which this FTQP point occurs is the critical value for the flow transition and it is called CNPR.

The series of snapshots in Fig. 6 shows the evolution of flow transition starting from the FSS flow regime to the RSS flow regime. With the evolution of time, the FTQP turns into two separate TP. The small normal shock that joins these two TP is called the annular MD (AMD). The upper part of the AMD makes a triple point with the incident and the reflected shock, while the lower part does so with the internal shock and the MS. Later on, this AMD turns into a reflected oblique shock for the internal shock and the MS interaction at the TP. At the same time, the slip line originating from this TP is inclined away from the nozzle axis, towards the nozzle wall. The streamlines deflect away from the nozzle axis in the region of this interaction. Previously, in FSS flow configuration, it has been observed that these streamlines seem to reflect back to the nozzle axis from the TP due to the Mach reflection. A conical reflected shock wave (also known as cone-shaped shock) from the triple point interacts with the incident shock and makes a quadruple point with the subsequent formation of two reflected oblique shocks. Finally, the complicated axisymmetric "cap-shock pattern" is seen which is a combined feature of the MS, cone-shaped 

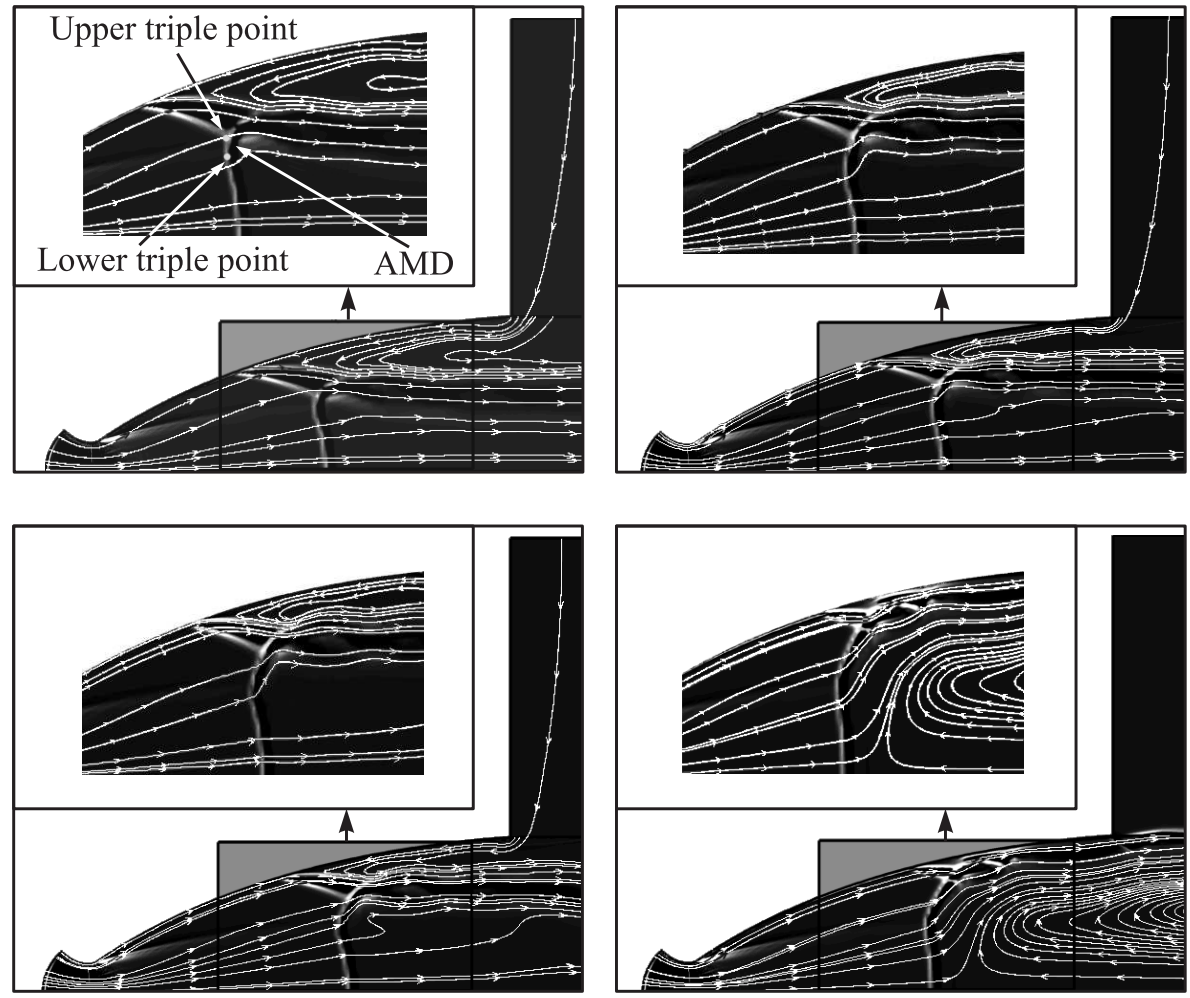

Figure 6 Evolution of flow structure during flow transition (FSS $\longrightarrow$ RSS)

shock, and the incident oblique shock due to the flow separation, also shown in Fig. 2.

During this process, pressure at the nozzle axis increases, and flow deflection away from the nozzle gives rise to the vortical structures along the nozzle axis. A negative axial momentum (against the main flow stream) resists the movement of the MS along the nozzle axis. Hence, the distance between the separation point and the location of the MS in the streamwise direction decreases. Consequently, the size of the incident shock relatively decreases and, on the other hand, the increase in the reflected shock from the TP can be seen. The resulting radial momentum away from the nozzle axis increases and diverts the flow towards the nozzle wall. Meanwhile, a vortical structure appears downstream of the cap-shock pattern. The size of this vortical structure increases with the increase in the size of cone-shaped shock and finally combines into one large trapped stabilized vortex of an adverse pressure gradient. The annular separated supersonic separated jet reattaches back to the nozzle wall and 


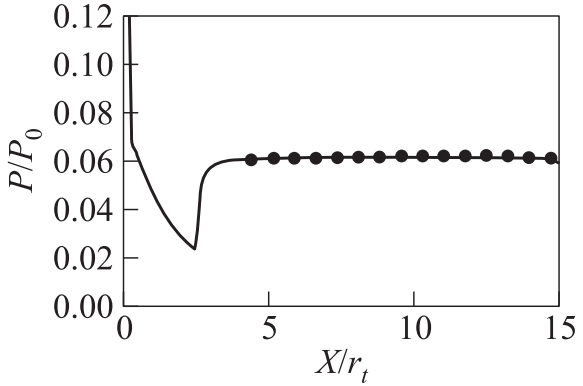

(a)

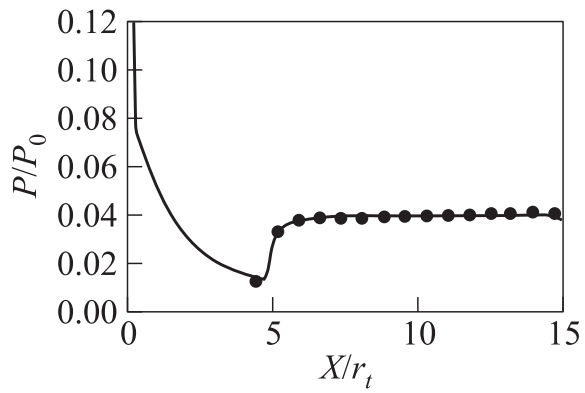

(c)

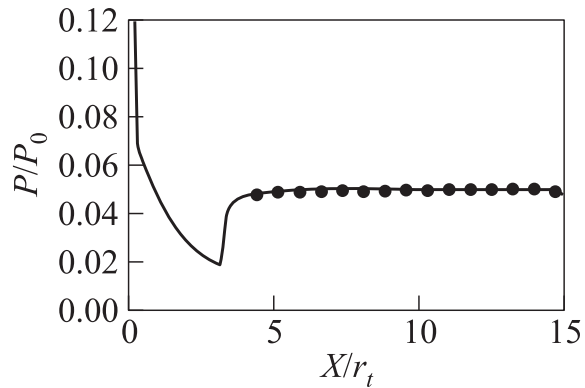

(b)

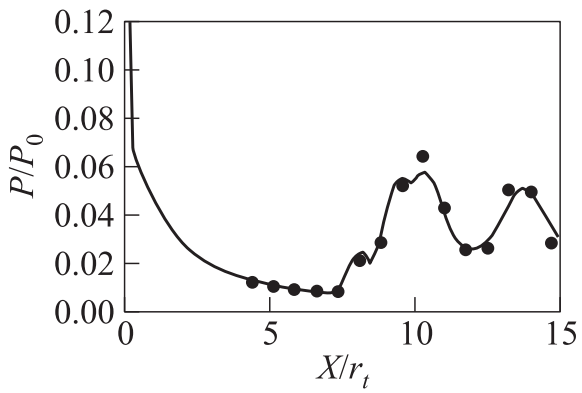

$(d)$

Figure 7 Evolution of mean pressure along the nozzle wall (curves) in comparison with experimental data [14] (signs): $(a) \mathrm{NPR} \approx 15.5 ;(b) 18.9 ;(c) 22.8$; and $(d) \mathrm{NPR}$ $\approx 25$

traps a small recirculation zone between the separation and the reattachment points. Finally, a very complex restricted shock separated flow regime can be seen.

Pressure evolution along the nozzle wall is different for FSS and RSS flow regimes. Numerically obtained wall pressure profiles at different NPR during this transition are compared with the available experimental data [14]. Figure 7 shows the plots of the wall pressure (normalized with the inlet stagnation pressure $P_{0}$ ) along the nozzle axis (normalized with the throat radius $r_{t}$ ). Numerically obtained wall pressures are in excellent agreement with their corresponding experimental data. It is seen that for FSS flow regime, the wall pressure suddenly increases at the incipient separation point and reaches a plateau which gradually increases but always remains lower than the ambient pressure. Whereas in the case of RSS flow regime after reaching the plateau, a sudden rise in the pressure occurs due to the reattachment of the flow. Further decrease and increase in the wall pressure are due to the successive interaction of shock and expansion waves in the annular supersonic jet. 
Based on these correctly predicted separation point (SP) locations, their relative distance with respect to the MS is plotted against the nozzle pressure ratio in Fig 8. Starting from lower NPR (say, 15), this relative distance of SP and MS increases and reaches its maximum at $\mathrm{NPR} \simeq 19$ in FSS mode. With further increase in the value of NPR towards the CNPR, this relative distance decreases. Once NPR $=24$ is reached (i.e., CNPR for LEATOC nozzle), a huge decrease in this difference has been observed which is

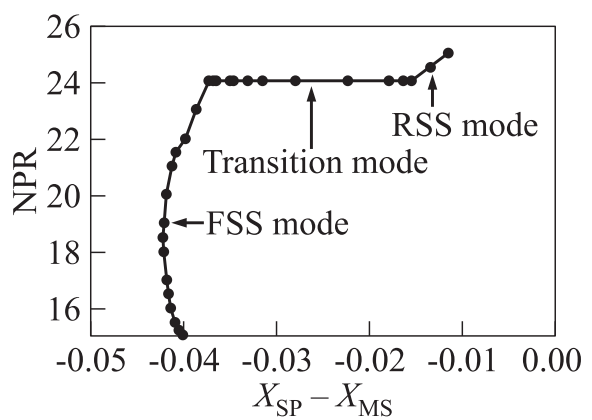

Figure 8 Evolution of relative distance between separation point and Mach stem w.r.t the NPR

due to the rapid movement of SP towards the nozzle exit w.r.t the slow moving MS in this very transition zone. Furthermore, in RSS mode, a linear decrease in its value near to zero is seen. Recently, Shams and Comte [19] in their detailed 3D numerical investigations on RSS flow regime have shown that for higher NPR, this SP moves downstream of the MS.

\section{EVOLUTION OF THE RESTRICTED SHOCK SEPARATION REGIME}

\subsection{Numerical Strategy}

Similar to the previous calculations, TGNS3D code has also been used to perform 3D numerical simulations to understand the behavior of RSS flow regime in LEATOC nozzle. For this purpose, a wide range of NPR, i. e., 25.5, 30, 38, 41.6, and 46, with stabilized inflow conditions, are extensively investigated and are reported in this part of the paper.

The grid used in these computations has been obtained by the extrusion of two-dimensional (2D) grid type $B$ with 72 grid points in the azimuthal direction, i. e., every $5^{\circ}$ around the nozzle axis. The whole $3 \mathrm{D}$ computational domain is composed of 4.032.000 grid points with 1.73 million nodes inside the nozzle.

A fifth-order monotonicity preserving weighted essentially nonoscillatory (MPWENO) numerical scheme [27] is used to achieve high order and phase accuracy results in such a complex flow in combination with a second order implicit scheme for time discretization. All simulations are performed by using newly proposed Detached Eddy Simulation (DES) [20]. This DES approach has shown results in good agreement with the experimental data for shock-separated flows in rocket nozzles. 


\subsection{Flow Physics}

In rocket nozzles with conical and thrust optimized (TO) contours, an internal shock is generated near the throat region. In parabolic and TO contour nozzles, this internal shock interacts with the small normal shock far away from the throat. A TP exists where the internal shock, the MS, and the cone-shaped oblique shock meet, indicating a connection between the internal shock and the existence of a cap-shock pattern which is responsible for the flow reattachment (Fig. 9).

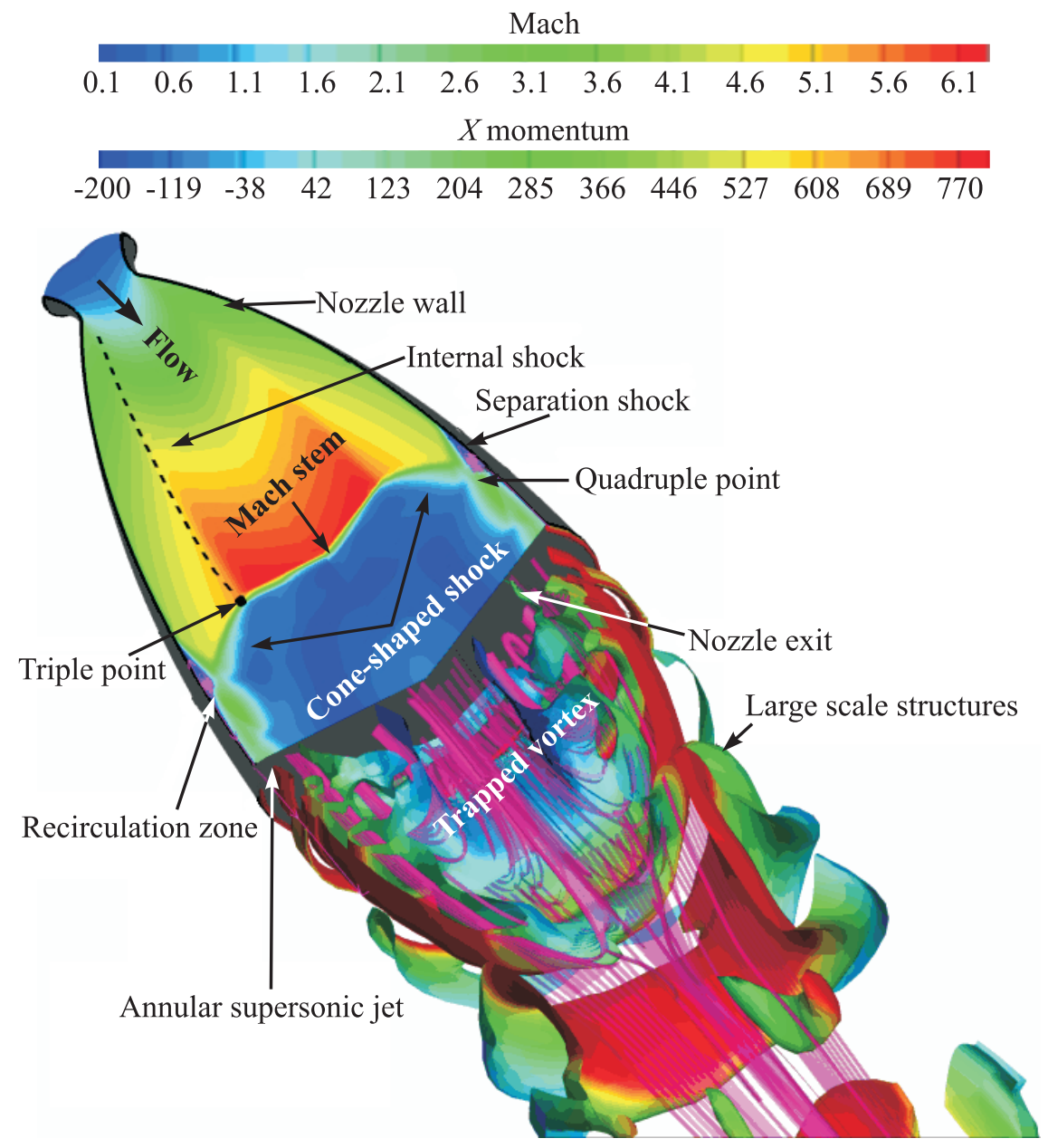

Figure 9 Restricted shock separation flow regime. (Refer Shams et al., p. 182.) 
In conical nozzles, although there exists an internal shock, these various internal shock reflections at the centerline destroy the high Mach number flow, and the Kernel is closed shortly downstream of the throat. Therefore, neither any cap-shock pattern nor reattachment of the flow are seen. In contrast, in Truncated Ideal Contoured (TIC) nozzles, no such internal shock exists and only free shock separated flows in TIC types nozzle are seen. In the recent past, the appearance of restricted shock separated flow has been reported in a Compressed Truncated Perfect nozzle [16]. However, they were unable to detect the internal shock due to the pattern of iso-Mach contours but have reported some low expansion region near the Mach reflection and suddenly the inverse Mach reflection is seen.

From the above discussion, one can conclude that this reattachment of the separated flow depends on the nozzle contour in a defined range of NPR. Figure 9 shows the successfully reproduced restricted shock separated flow regime at $\mathrm{NPR}=38.0$. A large stabilized trapped vortex downstream of the cap-shock pattern is shown with the help of $Q$-criterion coloured with momentum in the $x$-direction and streamlines. As depicted from Fig. 9, RSS flow regime exhibits a number of flow discontinuities which are a bit difficult to differentiate or even detect (e.g., internal shock) with iso-Mach contours. Therefore, the following shock function [22] was defined:

$$
f(x) \equiv \frac{\mathbf{U}}{c} \frac{\operatorname{grad} p}{|\operatorname{grad} p|} .
$$

Figure 10 shows the isocontours of shock function and one can see the clear vision of flow discontinuities, internal shock, cap-shock triple, QP, etc. The
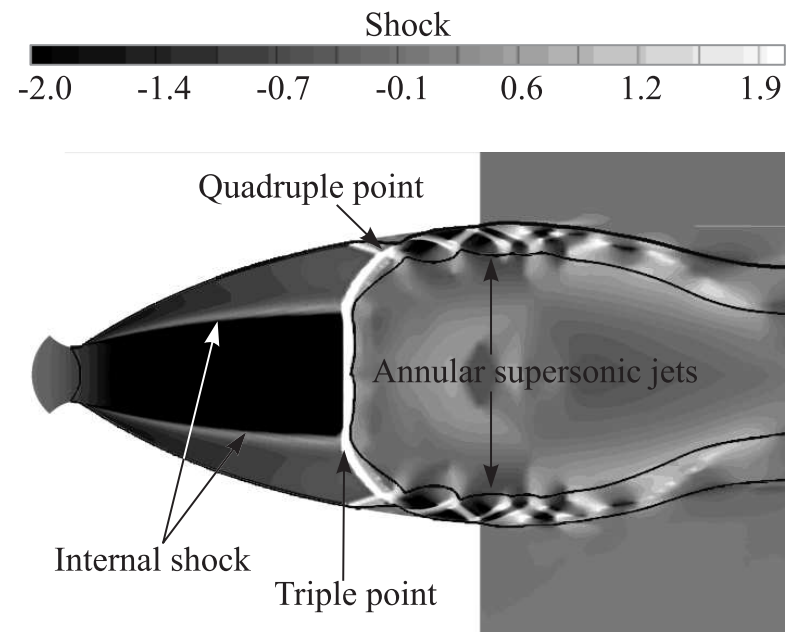

Figure 10 Isocontours of shock function $f(x)$ 
dark regions (negative value of the shock function) indicate the expansion in the flow and bright (positive value) zones highlight the compression regions. The annular supersonic jet which reattaches back to the nozzle wall exhibits successive interactions of shock and expansion waves, which sometimes leads to secondary flow separation which is shown in the following section. For more details about RSS flow regime see [20].

\subsection{Evolution of Wall Pressure}

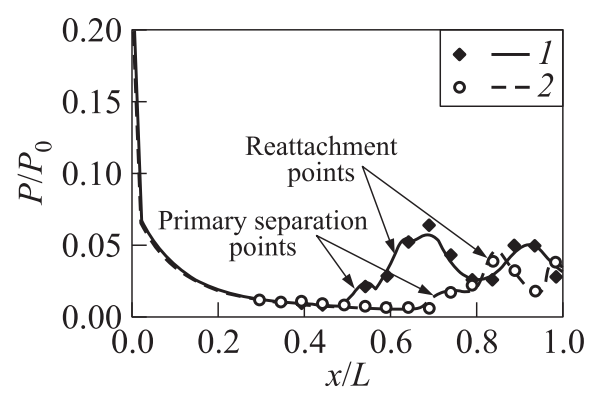

Figure 11 Evolution of mean wall pressure (curves) in comparison with experimental data $[14]$ (signs): $1-\mathrm{NPR} \approx 25.5$; and $2-\mathrm{NPR} \approx 38$ reattaches back to the nozzle wall, it makes the pressure increase even higher than the ambient pressure. The annular supersonic flow is subjected to expansion and compression waves reflected between the nozzle wall and the mixing layer separating the high-speed region from the central trapped vortex. These reflected waves give an oscillatory wall pressure distribution which can lead, in some cases, to secondary separation shown by Nguyen [13].

\subsection{Separation Point and Mach Stem Location}

An increase in nozzle pressure ratio causes the SP and corresponding MS to move further downstream (towards the nozzle exit), whatever the flow configuration is, FSS or RSS. In the case of FSS flow regimes, the respective distance between the SP and MS remains almost constant. Whereas in RSS flow regime, this is not the case, although at the initial stages (say, NPR $\geq 24$, when flow is in RSS zone), the respective distance between SP and the MS is quite small as compared to the FSS regime. Table 2 shows the numerically observed positions of the SP, MS, and their respective QP along with their corresponding NPRs. 
One can notice that for NPR $=25.0$, relative difference between Mach stem and separation point locations is positive, which is equal to 0.9 times the throat radius. This suggests that the Mach stem is located downstream of the separation point location. With the increasing NPR (say for 30.0) this difference decreases and even becomes negative for NPR $=38.0$. This means that the movement of the separation point location with respect to the Mach stem increases for higher NPR (i. e., 41.0, 46.0).

\subsection{Behavior of Recirculation Zones}

Apparently, two different recirculation zones appear in RSS flow regime: $(i)$ a recirculation bubble, which is trapped between separation and reattachment of supersonic jet; and (ii) a trapped vortex, large stabilized adverse pressure recirculation zone behind the cap-shock pattern.

Based on the unsteady flow behavior, the minimum and maximum ranges of these recirculation zones are given in Tables 3 and 4, respectively, against their corresponding NPRs. The overall range of the recirculation bubble varies from 1.69 to 2.33 times of the nozzle throat radius. The size of these recirculation zones is directly linked with the separation and reattachment point locations. It is shown in the previous section that the respective difference between separation and reattachment point locations increase with the increase in NPR, also depicted from results in Table 3.

The same kind of interpretation holds for the stabilized trapped vortex which appears just downstream of the cap-shock. The respective movement of Mach

Table 3 Evolution of minimum and maximum range of recirculation bubble with the increase in NPR

\begin{tabular}{ccc}
\hline \multirow{2}{*}{ NPR } & \multicolumn{2}{c}{$\Delta X / r_{t}$} \\
\cline { 2 - 3 } & Minimum & Maximum \\
\hline 25.0 & 1.69 & 1.97 \\
30.0 & 2.03 & 2.17 \\
38.0 & 2.16 & 2.29 \\
41.0 & 2.19 & 2.30 \\
46.0 & 2.24 & 2.33 \\
\hline
\end{tabular}

Table 4 Evolution of minimum and maximum range of trapped vortex with the increase in NPR

\begin{tabular}{ccc}
\hline \multirow{2}{*}{ NPR } & \multicolumn{2}{c}{$\Delta X / r_{t}$} \\
\cline { 2 - 3 } & Minimum & Maximum \\
\hline 25.0 & 1.32 & 1.48 \\
30.0 & 1.33 & 1.51 \\
38.0 & 1.34 & 1.53 \\
41.0 & 1.38 & 1.61 \\
46.0 & 1.41 & 1.65 \\
\hline
\end{tabular}


stem towards the nozzle exit with the increase in NPR causes an increase in the size of the trapped vortex, ranging from 1.32 (minimum) to 1.65 (maximum) times of the nozzle exit radius. The difference between the minimum and maximum range of this trapped vortex shows an increase for higher NPR, which indicates that for higher NPR, a high level of fluctuations appears.

\subsection{Behavior of Axial and Radial Momentum}

In the RSS flow regime, an internal shock from the throat region interacts with the Mach and forms a TP with a cone-shaped shock (see Fig. 9). A slip line emanates from this TP, which is inclined away from the nozzle axis and diverts the flow towards the nozzle wall due to the increasing radial momentum. To understand this phenomenon, radial momentum profiles (from nozzle axis to wall) before and after MS are shown in Fig. 12.

The upstream location (before MS) for radial momentum distribution w.r.t the MS is $\Delta x / r_{t}=0.6$. The downstream position is selected as the quadru-

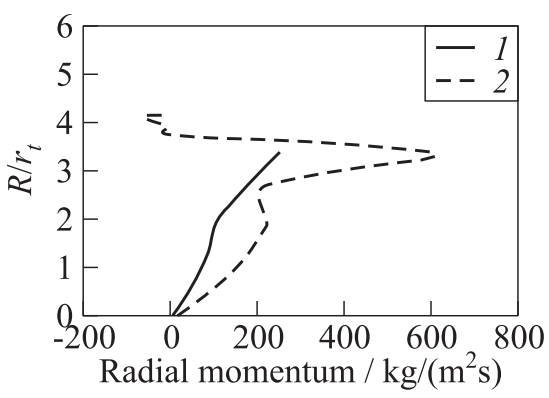

(a)

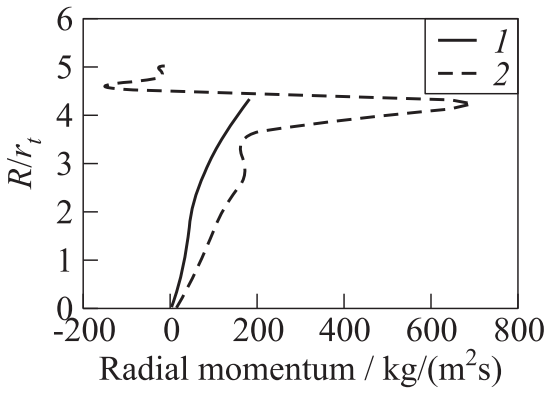

(c)

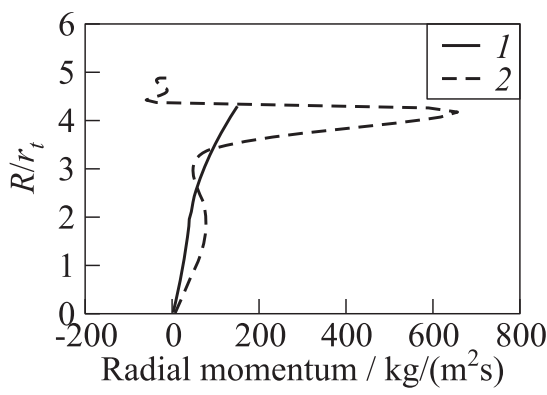

(b)

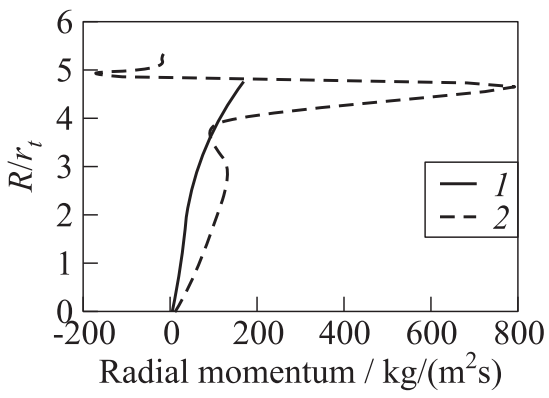

(d)

Figure 12 Distribution of radial momentum before (1) and after (2) MS for $\mathrm{NPR}=25.0(a), 38.0(b), 41.0(c)$, and $46.0(d)$ 


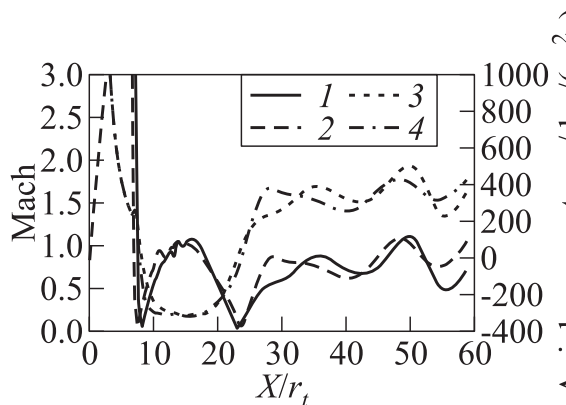

(a)

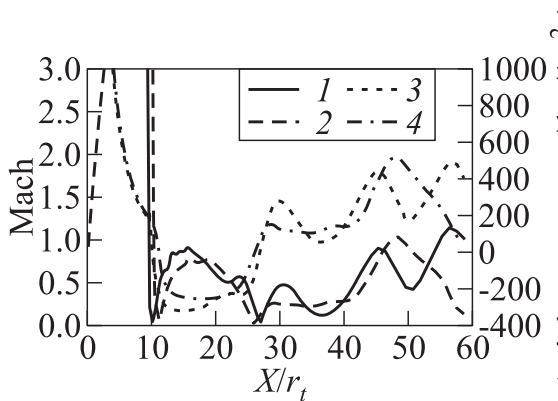

(a)

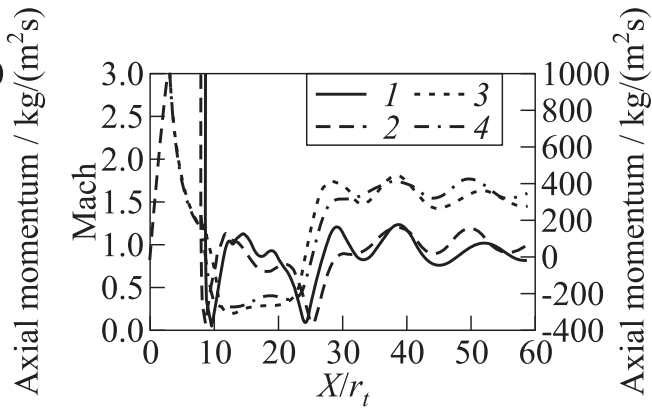

(a)

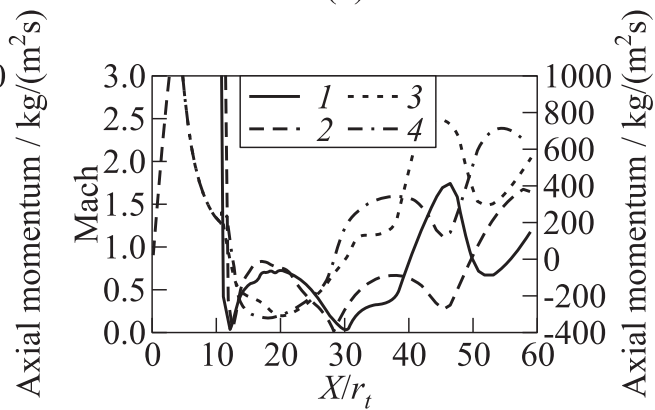

(a)

Figure 13 Evolutions of Mach number and axial momentum along the nozzle axis for NPR $=25.0(a), 38.0(b), 41.0(c)$, and $46.0(d)$

ple point location observed against the mentioned NPRs. Radial momentum distribution before the MS shows the positive value because of the expansion region. Downstream of the MS, value of radial momentum increases slowly from zero (nozzle axis) on the positive side. Near the TP region, it shows a sudden increase in the value of radial momentum and reaches its maximum at the quadruple point. Above the quadruple point, radial momentum decreases and turns into negative zone (momentum towards nozzle axis) caused by a small recirculation zone and finally falls at the wall. The range of the radial momentum on both the positive and negative sides increases with the increase in the NPR. This is due to the fact that relative size of recirculation bubble and cone-shaped shock increase for higher NPR.

With this increase in positive radial momentum downstream of MS, pressure along the nozzle axis increases and produces a negative force against the main flow. This negative force in terms of axial momentum along the nozzle is shown in Fig. 13. Axial momentum and Mach number distributions seem to be the right detector for the size of the trapped vortex (also shown in Table 4). Figure 13 shows that the minimum values of Mach number correspond to the locations 
where axial momentum turns from positive to negative values, indicating the range of the trapped zone.

Increase in the NPR (chamber pressure) causes the movement of cap-shock pattern towards the nozzle exit. The position of the MS is determined by a balance between the positive axial momentum just downstream of the MS (before the stagnation point) and a negative axial momentum in the trapped vortex (after the stagnation point). Whereas in the outer part of cap-shock pattern, there is a large amount of positive axial momentum corresponding to the supersonic flow regime. The position of the separation point is determined by the level of pressure in the recirculation bubble. As it was shoen, the difference between the locations of MS and the SP along the nozzle axis goes from positive to negative. As a consequence of this movement, the length of the cone-shaped shock relatively increases whereas the length of the separation shock decreases. The resulting radial momentum away from the nozzle axis relatively increases.

\section{CONCLUDING REMARKS}

Numerical simulations have been performed to understand the formation of the cap-shock pattern and the behavior of restricted shock separated flow regime appearing in a TOC nozzle when operating under overexpanded conditions.

In the first part of the paper, the flow transition from FSS to RSS has been reproduced by axisymmetric calculations and it was found to be in good agreement with the experiments (see section 4). The main goal of this study was to understand the phenomenon of the cap-shock formation. This complicated pattern appears only in the nozzles which exhibit an internal shock near the throat region. The present study has shown that formation of the cap-shock pattern is triggered when internal and separated shocks interact with the MS and form two reflected shocks at a common interaction point. This common interaction point (which is denoted as FTQP) occurs corresponding to its CNPR, which is 24 for the LEATOC nozzle. As a consequence of cap-shock pattern, radial momentum away from the nozzle increases and causes the separated annular supersonic jet to reattach back to the nozzle wall.

In the second part of the paper, a 3D numerical investigation of restricted shock separated flow has been performed (see section 5). In the LEATOC nozzle, the appearance of RSS flow regime started for NPR $\geq 24$. Simulations were performed at five different NPRs ranging from 25 to 46 . With the increase in NPR (within the RSS range), SP locations move from upstream to downstream of the MS. As a consequence, the size of the cone-shaped shock increases. This movement of separation point location also causes the increase in the size of the recirculation zone trapped between the separation and reattachment of the annular supersonic jet. On the other hand, the relative size of the stabilized 
trapped vortex downstream of the cap-shock pattern increases with the NPR. Negative axial momentum distribution along the nozzle axis downstream of the cap-shock pattern produces a push against the main flow, and is one of the possible sources due to which SP locations shift downstream of the MS. Radial momentum distribution across the respective QP increases with the NPR, which bolsters up the above interpretation.

\section{ACKNOWLEDGMENTS}

The Higher Education Commission (HEC), Government of Pakistan, is acknowledged for the research grant. The first author would like to thank T. Alziary de Roquefort for many discussions related to this research work.

\section{REFERENCES}

1. Summerfield, M., C. Foster, and W. Swan. 1954. Flow separation in overexpanded superonic exhaust nozzles. Jet Propul. 319.

2. Nave, L. H., and G. A. Coffey. 1973. Sea-level side-loads in high area ratio rocket engines. AIAA Paper No. 73-1284.

3. Chen, C. L., S. R. Chakravarthy, and C. M. Hung. 1994. Numerical investigation of separated nozzle flows. AIAA J. 32:1836-43.

4. Frey, M., and G. Hagemann. 1998. Status of flow separation prediction in rocket nozzles. AIAA Paper No. 98-3619

5. Frey, M., and G. Hagemann. 1999. Flow separation and side-loads in rocket nozzles, AIAA Paper No. 99-2815.

6. Moore, J. G., and J. Moore. 1999. Realizability in two equation models. AIAA Paper No. 99-3779.

7. Frey, M., and G. Hagemann. 2000. Restricted shock separation in rocket nozzles. J. Propul. Power 16(3).

8. Girard, S., H. Deniau, A.T. Nguyen, and T. Alziary de Roquefort. 2001. Etude de l'écoulement dans une tuyére propulsive à contour parabolique en régime surdétendu. 37eme Colloque d'Aérodynamique Appliquée de l'AAAF: Aérodynamique et Propulsion des Véhicules à grande vitesse. Aracachon, France.

9. Deck, S. 2002. Simulation numériques des charges latérale instationaires sur des configuration de lanceurs. Ph.D. Thesis. Université d'Orléans.

10. Deck, S., E. Garnier, and P. Guillen. 2002. Turbulence modelling applied to space launcher configurations. J. Turbulence 3:057.

11. Hagemann, G., M. Frey, and W. Koschel. 2002. Appearance of restricted shock separation in rocket nozzles. J. Propul. Power 18(3).

12. Pilinski, C. 2002. Etude numérique du decollément en tuyére supérsonique. Ph.D. Thesis. INSA de Rouen. 
13. Nguyen, A. T. 2003. Découlement instationaire et charges latérales dans les tuyéres propulsives. Ph.D. Thesis. Université de Poitiers.

14. Nguyen, A. T., H. Deniau, S. Girard, and T. Alziary de Roquefort. 2003. Unsteadiness of flow separation and end-effects regime in a thrust-optimized contour rocket nozzle. Flow Turbulence Combust. 71:161-81.

15. Deck, S., and A. T. Nguyen. 2004. Unsteady side loads in a thrust-optimized contour nozzle at hysteresis regime. J. Propul. Power 42(9):1878-88.

16. Shimizu, T., M. Hiroshi, and K. Masatoshi. 2006. Numerical study of restricted shock separation in a compressed truncated perfect nozzle. AIAA J. 44(3).

17. Hagemann, G., and M. Frey. 2008. Shock pattern in the plume of rocket nozzles: Needs for design consideration. Shock Waves 17:387-95.

18. Morínigo, J. A., and J. J. Salvá. 2008. Numerical study of the start-up process in an optimized rocket nozzle. J. Aerospace Sci. Technol. 12:485-89.

19. Shams, A., and P. Comte. 2008. Status of restricted shock separation in rocket nozzles. 3rd European Conference for Aerospace Sciences (EUCASS). Versailles, France.

20. Shams, A., P. Comte, S. Girard, G. Lehnasch, and M. F. Shahab. 2008. 3D unsteady numerical investigation of an overexpanded thrust optimized contour nozzle. 6th European Symposium on Aerothermodynamics for Space Vehicles. Versaille, France.

21. Reijasse, P., F. Bouvier, and P. Servel. 2002. Experimental and numerical investigation of the cap-shock structure in over-expanded thrust-optimized nozzles. WestEast High Speed Flow Field Conference. Marseille, France.

22. Lovely, D., and R. Haimes. 1999. Shock detection function from computational fluid dynamics results. AIAA Paper No. 99-3285.

23. Rao, G. V.R. 1961. Recent developments in rocket nozzle configurations. ARS J. 31(11):1488-94.

24. Poinsot, T. J., and S. K. Lele. 1992. Boundary conditions for direct simulations of compressible viscous flows. J. Comput. Phys. 101:104-29.

25. Rudy, D. H., and J. C. Strikwerda. 1981. A non-reflecting outflow boundary condition for subsonic Navier-Stokes calculations. J. Comput. Phys. 36:55-70.

26. Wilcox, D.C. 1988. Reassessment of the scale deterring equation for advanced turbulence models. AIAA J. 26:1299-310.

27. Balsara, D. S., and C. W. Shu. 2000. Monotonicity preserving weighted essentially non-oscillatory schemes with increasing high order of accuracy. J. Comput. Phys. 160:405-52. 